\title{
The link between CME-associated dimmings and interplanetary magnetic clouds
}

\author{
Cristina H. Mandrini ${ }^{1}$, María S. Nakwacki ${ }^{1}$, Gemma Attrill ${ }^{2,6}$, Lidia \\ van Driel-Gesztelyi ${ }^{2,3,4}$, Sergio Dasso ${ }^{1,5}$ and Pascal Démoulin ${ }^{3}$ \\ ${ }^{1}$ Instituto de Astronomía y Física del Espacio, CC. 67 Suc. 28, 1428, Buenos Aires, Argentina \\ email: mandrini@iafe.uba.ar \\ ${ }^{2}$ University College London, Mullard Space Science Laboratory, \\ Holmbury St.Mary, Dorking, Surrey, RH5 6NT, UK \\ ${ }^{3}$ Observatoire de Paris, LESIA, UMR 8109 (CNRS), 92195 Meudon Principal Cedex, France \\ ${ }^{4}$ Konkoly Observatory of the Hungarian Academy of Sciences, Budapest, Hungary \\ ${ }^{5}$ Departamento de Física, Facultad de Ciencias Exactas y Naturales, \\ Universidad de Buenos Aires \\ ${ }^{6}$ Harvard-Smithsonian Center for Astrophysics, 60 Garden St., Cambridge, MA 02138, USA
}

\begin{abstract}
Coronal dimmings often develop in the vicinity of erupting magnetic configurations. It has been suggested that they mark the location of the footpoints of ejected flux ropes and, thus, their magnetic flux can be used as a proxy for the ejected flux. If so, this quantity can be compared to the flux in the associated interplanetary magnetic cloud (MC) to find clues about the origin of the ejected flux rope. In the context of this interpretation, we present several events for which we have done a comparative solar-interplanetary analysis. We combine SOHO/Extreme Ultraviolet Imaging Telescope (EIT) data and Michelson Doppler Imager (MDI) magnetic maps to identify and measure the flux in the dimmed regions. We model the associated MCs and compute their magnetic flux using in situ observations. We find that the magnetic fluxes in the dimmings and MCs are compatible in some events; though this is not the case for large-scale and intense eruptions that occur in regions that are not isolated from others. We conclude that, in these particular cases, a fraction of the dimmed regions can be formed by reconnection between the erupting field and the surrounding magnetic structures, via a stepping process that can also explain other CME associated events.
\end{abstract}

Keywords. Sun: coronal mass ejections (CMEs), Sun: magnetic fields, interplanetary medium

\section{Introduction}

The plasma and magnetic field ejected from the Sun by coronal mass ejections (CMEs) are later observed in the interplanetary (IP) medium as interplanetary coronal mass ejections (ICMEs). When certain characteristics are present (low plasma $\beta$, lower proton temperature and stronger magnetic field than in the ambient solar wind, exhibiting a smooth and significant rotation), ICMEs are called magnetic clouds (MCs) (Burlaga et al. 1981). Several studies relating qualitatively and/or quantitatively MCs with their solar sources have been published in recent years (see the review by Démoulin 2007).

The two global MHD invariants used to link coronal to IP observations are the magnetic field helicity and flux. Magnetic helicity, at the solar level and in the IP medium, can be derived from observations combined with magnetic field models. At the solar level the ejected helicity can be estimated from the helicity decrease of the coronal field after an ejection, this value in general agrees with the helicity contained in the associated $\mathrm{MC}$ (see Mandrini et al. 2007 and references therein). 
Concerning the ejected magnetic flux, we have to use proper proxies to estimate it. Two solar proxies have been used so far. First, the photospheric flux of the dimming regions forming after CMEs (see Mandrini et al. 2007 and references therein) and, second, the one swept out by flare ribbons as they separate, moving away from the magnetic inversion line (see Qiu et al. 2007 and references therein). How do these features relate to the ejected flux? What can we learn from the comparison between the estimated ejected flux and the flux in the associated MC when considering both proxies?

In general, coronal dimmings are interpreted as density depletions caused by the eruption of an unstable magnetic configuration. The eruption leads to the expansion of magnetic loops and the evacuation of plasma along them into the IP space (Hudson et al. 1996). Two dimmings (primary dimmings) are often seen to form on both sides of the erupting configuration. Two physical models have been proposed. In a first case, dimmings correspond to the footpoints of the ejected flux rope, as suggested by Webb et al. (2000). In this context, the flux rope pre-exists in the corona and remains rooted in the dimming regions, as it expands out into the IP. The flux of the dimming regions is comparable to the flux in the axial magnetic field component of the MC in this case. In a second case, the arcade above the flux rope expands significantly before reconnecting (below the flux rope). Dimmings appear at the footpoints of the flux rope and also all along the footpoints of the sheared magnetic arcade. As reconnection proceeds, more flux is progressively added to the erupting flux tube (see next paragraph). In this second case, that corresponds to the theoretical two-dimensional model of Lin \& Forbes (2000), the flux in the dimming regions is comparable to the sum of the flux in the axial and azimuthal components of the MC. If the flux rope does not already exist in the corona in this second case, it will be formed during the reconnection process.

The magnetic flux swept out by flare ribbons, a proxy for the reconnected flux, can also be used, as follows (see Qiu et al. 2007). In a classical flare model, magnetic reconnection occurs mainly below a pre-existing flux rope (see Lin \& Forbes 2000 and references therein). The magnetic flux forming 'post'-flare loops is identical to the flux added to the pre-existing flux rope that will be observed in the IP space as an MC. Then, the flux in the azimuthal component of the MC field will be comparable (if the ejected flux rope was mostly formed by reconnection during the ejection) or larger (when the flux rope was present before ejection) than the flux swept out by flare ribbons.

The above discussion shows that the comparison of coronal and IP associated events can be used to constrain the CME mechanism; in particular, in relation to determining whether the ejected flux rope was pre-existing in the corona, or whether it formed during the eruption. In this paper we present an overview of examples previously analyzed (Section 2). These are: a minor eruptive event on 11 May 1998 (see Mandrini et al. 2005, Paper I), a long duration C1.3 class flare and CME (see Attrill et al. 2006, Paper II) on 12 May 1997, and the large X17 flare and CME on 28 October 2003 (see Mandrini et al. 2007, Paper III). Comparison of these examples allows us to discuss under which circumstances either (i) the magnetic flux in the dimmings or (ii) the magnetic flux swept out by the flare ribbons, may be used as reliable proxies for the ejected flux (Section 3).

\section{Overview of three events with different characteristics}

The image on the left panel of Fig. 1 shows an elongated feature at disk center. This small, isolated, bipolar and non-numbered active region (AR) showed an eruptive nature during 11 May 1998. The largest event at 08:31 UT was accompanied by the elongation of the sigmoidal AR loops that later disappeared, EUV dimmings and cusp formation. The dimmings were located at both sides of the AR, lying partially over each polarity and 


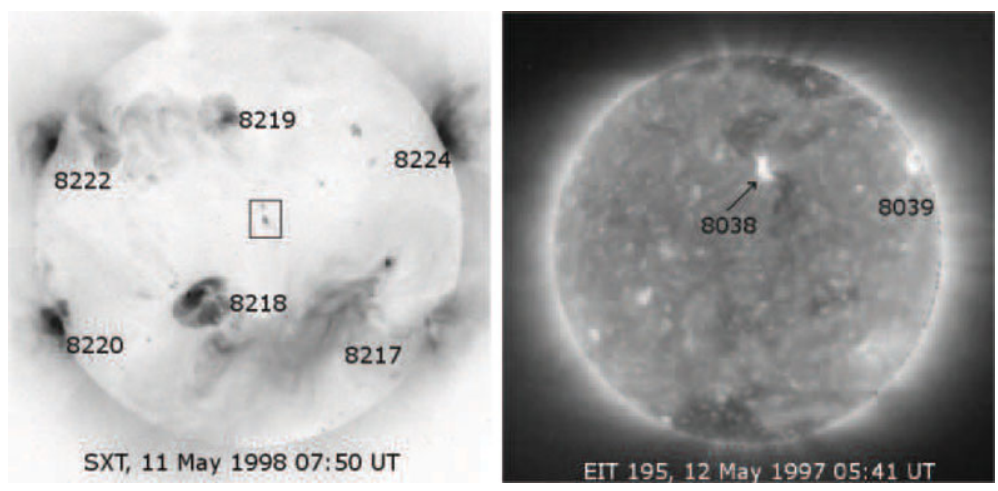

Figure 1. Left: Soft X-ray Telescope (Yohkoh/SXT) image (reversed color) at the maximum extension of the bright loops in the small eruptive AR (within the box) located far from other ARs. Right: EIT image showing the two dimmings on both sides of the isolated AR 8038 .

extending into the nearby quiet Sun regions. Following the procedure discussed in Paper $\mathrm{I}$, we found that the absolute value of the total net flux in each dimming was $\mathrm{F}_{\text {dim }}=$ $(13 \pm 2) \times 10^{19} \mathrm{Mx}$, meaning that the flux in these primary dimmings was balanced. Analyzing in situ data from the Wind satellite, we were able to identify the resulting tiny MC. We modeled the magnetic data using a cylindrical linear force-free static model (see Paper I) and determined the axial $\left(\mathrm{F}_{z, \text { cloud }}\right)$ and azimuthal $\left(\mathrm{F}_{y, \text { cloud }}\right)$ fluxes, considering a cloud length constrained by solar and IP observations. These values were: $\mathrm{F}_{z, \text { cloud }}=$ $1.3 \times 10^{19} \mathrm{Mx}$ and $10 \times 10^{19} \mathrm{Mx} \leqslant \mathrm{F}_{y, \text { cloud }} \leqslant 20 \times 10^{19} \mathrm{Mx}$.

We proceeded in a similar way for the event shown in the right panel of Fig. 1. AR8038, which was mainly bipolar, was the only region on the solar disk at that time. A long duration C1.3 flare started at 04:42 UT, it was accompanied by a filament eruption, CME, coronal wave and dimmings (see Paper II and references therein). Two main dimmings were present at both sides of the AR lying again partially over the AR polarities and quiet Sun regions. Defining the dimming boundaries as discussed in Paper II, we computed a net flux of $\mathrm{F}_{\text {dim }}=(21 \pm 7) \times 10^{20} \mathrm{Mx}$ for the southern-most dimming at its maximum extension (the southern-most dimming was identified as the sole remaining footpoint of the erupted flux rope, since during the eruption the northern-most edge of the flux rope underwent an interchange reconnection with the north polar coronal hole, transferring its connectivity from the Sun to the interplanetary magnetic field). The MC associated to this eruption was observed by Wind. We modeled the magnetic data using three different cylindrical and static models (see Paper II) and found an axial $\mathrm{MC}$ flux $\mathrm{F}_{z, \text { cloud }}=$ $(4.8 \pm 0.8) \times 10^{20} \mathrm{Mx}$ and an azimuthal $\mathrm{MC}$ flux $\mathrm{F}_{y, \text { cloud }}=(17 \pm 8) \times 10^{20} \mathrm{Mx}$, taking a length constrained by solar and IP observations.

One of the largest "Halloween" events was the X17 flare on 28 October 2003 in AR 10486. These events occurred just after solar maximum when the Sun's field was highly complex (Fig. 2, right panel). The X17 flare was accompanied by a filament eruption, CME, coronal EIT and Moreton waves and very extended dimmings (Fig. 2, left panel). Following the method discussed in Paper III, we found for the signed net flux in the numbered dimmings: $\mathrm{F}_{\operatorname{dim} 1}=-4.4 \times 10^{21} \mathrm{Mx}, \mathrm{F}_{\operatorname{dim} 2}=0.7 \times 10^{21} \mathrm{Mx}, \mathrm{F}_{\operatorname{dim} 3}=1.7 \times 10^{21}$ $\mathrm{Mx}$, and $\mathrm{F}_{\text {dim } 4}=-7.0 \times 10^{21} \mathrm{Mx}$. We noticed that none of the dimmed regions lay on the AR main polarities. Though two main dimming regions were located to the west and two to the east of the AR, the net flux was dominantly negative on either side. Therefore, these dimmings were not of the same kind as the ones found in the previous examples. We concluded that primary dimmings were not observed in this case. This 

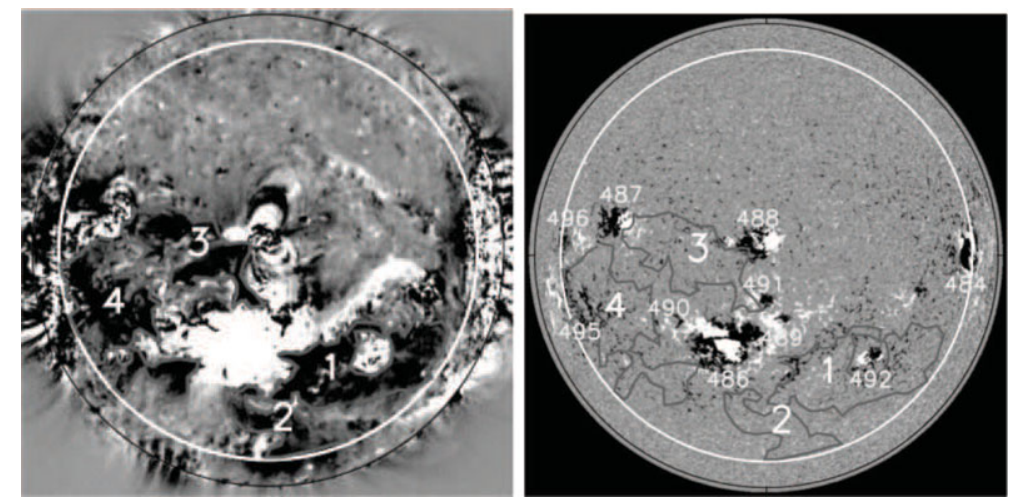

Figure 2. Left: EIT 195 base difference (12:00 UT - 05:00 UT) image showing the largest dimmed regions $(1,2,3$, and 4) around 1 hour after flare onset. The white circle has been drawn at an angular distance of $60^{\circ}$ from Sun center. MDI magnetic field measurements are done within this limit. Right: Dimmed regions overplotted on an MDI magnetogram at 11:11 UT. All the ARs present on the solar disk are identified (these numbers should start with 10).

happened because the major energy release during the flare was followed by strong chromospheric evaporation and formation of dense and bright loops that make the primary dimmings unobservable. Therefore, the observed distant and extended dimmings must be secondary; these dimmings can be formed by a process illustrated in Fig. 3, as follows. The expanding CME loops reconnect with favorably oriented loops to the west and east. This reconnection process creates two new sets of connectivities: small loops (that become bright due to evaporation) and large-scale loops belonging to the eruptive configuration with displaced footpoints. Dimmings are expected to be associated with these long loops as the plasma expands to fill a larger volume. The MC associated with this event was in strong expansion, so, we modeled its magnetic field using two expansion

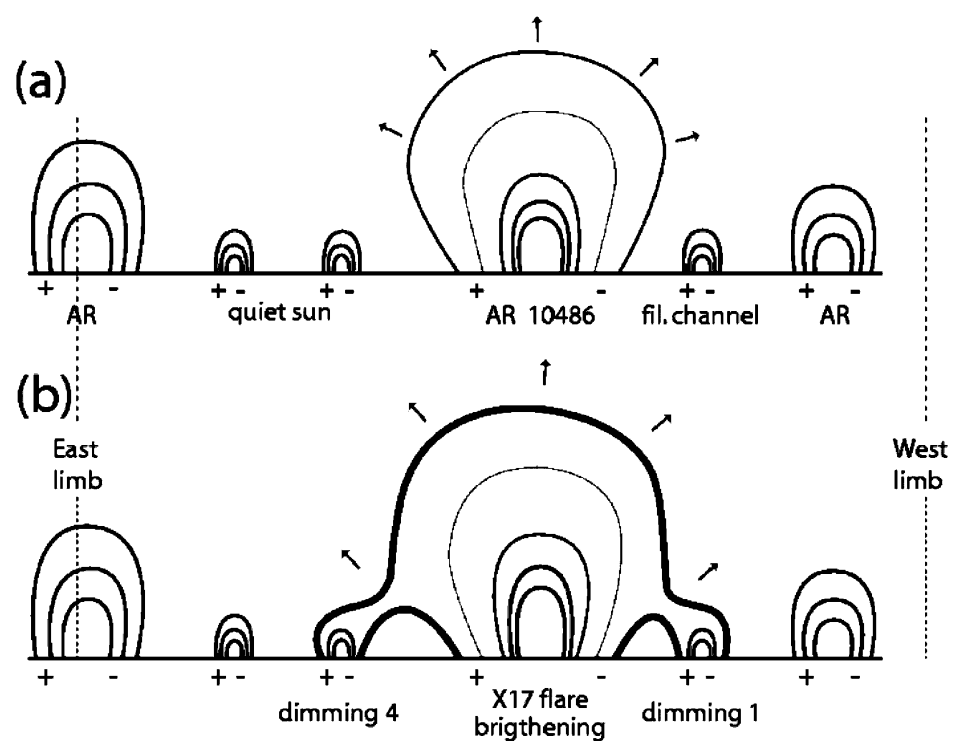

Figure 3. The expanding CME magnetic configuration (a) and its interaction (b) with the surrounding bipoles and ARs resulting in the spread of dimmings (same mechanism proposed by Attrill et al. 2007, see text). 
models as well as a static one for comparison (see Paper III). We found an axial MC flux $2.8 \times 10^{21} \mathrm{Mx} \leqslant \mathrm{F}_{z, \text { cloud }} \leqslant 3.1 \times 10^{21} \mathrm{Mx}$ and an azimuthal $\mathrm{MC}$ flux $11 \times 10^{21} \mathrm{Mx}$ $\leqslant \mathrm{F}_{y, \text { cloud }} \leqslant 16 \times 10^{21} \mathrm{Mx}$. Following our discussion on the formation of the dimmings for this event via secondary reconnections with the surrounding fields, it is therefore not possible to directly compare the flux in the MC to the flux in the dimmings. Qiu et al. (2007) have computed the flux swept out by flare ribbons for this event and found a value of $(18.8 \pm 1.8) \times 10^{21} \mathrm{Mx}$, which is in good agreement with the total MC flux.

\section{Discussion and conclusions}

In summary, in the three analyzed examples either the magnetic flux in the dimming regions or the one swept out by flare ribbons was found to be compatible primarily with the azimuthal MC flux. This indicates that the ejected rope was mostly formed by magnetic reconnection during the eruption process.

Qiu et al. (2007) found a different result concerning the flux in coronal dimming regions. These authors concluded that the flux in dimming regions is comparable in order of magnitude to the axial MC flux. However, in the common studied case, the 28 October 2003 X17 flare and CME, we have shown that the observed extended dimmings are secondary. The formation of these secondary dimmings can be explained via a stepping reconnection process, similar to the mechanism proposed by Attrill et al. (2007) for the bright front and diffuse leading edge of coronal waves. Although magnetic reconnection conserves magnetic flux, one cannot be sure how much of the magnetic flux in the secondary dimmings became part of the CME; therefore, these secondary dimming areas do not provide a proper proxy for magnetic flux measurements to be compared with interplanetary data. In fact, a detailed study of each event should be done before comparing the magnetic flux in dimmings to that of the associated MC.

Magnetic flux in coronal dimmings is a reliable proxy for the ejected flux in minor events (i.e. low X-class flares) that occur far from other flux concentrations. In these cases, primary dimmings may be observed. Furthermore, in such cases coronal dimmings can be the only useful proxy since it may not be possible to observe the evolution of flare ribbons or kernels. For large and very intense events, the flux swept out by flare ribbons is a more reliable proxy. We conclude that the most reliable solar proxy for the magnetic flux involved in an ejection depends upon the particular characteristics of the analyzed event.

\section{References}

Attrill, G., Harra, L. K., van Driel-Gesztelyi, L., \& Démoulin, P. 2007, ApJ, 656, L101.

Attrill, G., Nakwacki, M. S., Harra, L. K., van Driel-Gesztelyi, L., Mandrini, C. H., Dasso, S., \& Wang, J. 2006, Solar Phys. 238, 117.

Burlaga, L., Sittler, E., Mariani, F., \& Schwenn, R. 1981, Jour. Geophys. Res., 86, 6673.

Démoulin, P. 2007, An. Geo. 207, 87.

Hudson, H. S., Acton, L. W., \& Freeland, S. L. 1996, ApJ 470, 629.

Lin, J. \& Forbes, T. G. 2000, Jour. Geophys. Res. 105, 2375.

Mandrini, C. H., Nakwacki, M. S., Attrill, G., van Driel-Gesztelyi, L., Démoulin, P., Dasso, S., \& Elliott, H. 2007, Solar Phys., 244, 25.

Mandrini, C. H., Pohjolainen, S., Dasso, S., Green, L. M., Démoulin, P., van Driel-Gesztelyi, L., Copperwheat, C., \& Foley, C. 2005, A\&A, 434, 725.

Qiu, J., Hu, Q., Howard, T. A., \& Yurchyshyn, V. B. 2007, ApJ, 659, 758.

Webb, D. F., Lepping, R. P., Burlaga, L. F., DeForest, C. E., Larson, D. E., Martin, S. F., Plunkett, S. P., \& Rust, D. M. 2000, Jour. Geophys. Res., 105, 27251. 


\section{Discussion}

SCHMIEDER: Your cartoon about magnetic reconnection between the expanding magnetic configuration and the nearly magnetic shocks reminds me of the existence of the magnetic carpet with magnetic separatrices. The progression of these reconnections could explain the propagation of waves. On the other hand be careful with the flux measurements of MDI in dimmings.

MANDRINI: Of course this reconnection process can explain the progression of waves. Concerning your question about flux measurements, we have corrected MDI data for underestimation of flux, we have taken into account projection effects and none of the pixels in the dimming had saturated values. Besides all our flux measurements were done above a threshold of 20 Gauss.

VLAHOS: Reconnection is a small scale phenomenon but can have large scale re-arrangements of magnetic topologies. Large scale current sheets do not survive very long (milliseconds). So I prefer the phrase "large magnetic field re-arrangements caused by reconnection". Do you agree?

MANDRINI: Yes, I do.

WeBB: In reply to Vlahos comment: I think we have data on large-scale reconnection in current sheets trailing CMEs. We see ray-like structures following CMEs, evidence of reconnection region moving out, that can last for hours.

MALANDRAKI: Concerning the connectivity issue of the ICME observed in October 2003 you mentioned in the talk, I would like to draw your attention to the paper by Malandraki et al. 2005, J. Geophys, Res. Vol. 110, A09506, doi: 101029/2004JA010926, which presents near-relativistic electron observations from the EPAM experiment onboard ACE in the vicinity of and during the passage of the ICME over the s/c. The analysis of bi-directional near-relativistic electron pitch-angle distributions observed during the ICME along with the electron intensity characteristic provide strong evidence that loop-like IMF structures still anchored to the Sun are threading through this ICME. Furthermore, Malandraki et al., 2005 have also used the energetic particle observations by ACE/EPAM to identify more accurately the leading and trailing edge of this ICME compared to these previously identified. 